\title{
Schuldenerlass - quo vadis? Anreizmechanismen für eine nachhaltige Schuldensituation in Entwicklungsländern?
}

\author{
Von Stefan Hohberger, Bayreuth*
}

\section{Problemstellung}

"Debt relief has become the feel-good economic policy of the new millennium."1 So kommentiert Easterly die Entwicklungen in der Politik zum Thema „Schuldenerlass für Entwicklungsländer“. Die Frage nach einem Schuldenerlass für hoch verschuldete Länder ist ein vieldiskutiertes Thema und keine Neuheit auf der politischen Agenda. Bereits seit Ende der 1980er Jahre wurde mit Hilfe verschiedener Entschuldungsprogramme versucht, die externe Schuldenlast auf ein nachhaltiges Niveau zu senken. Eine Verringerung der Schulden - so die Hoffnung der Industrieländer - verbessere die finanzielle Situation der Schuldnerländer, führe zu vermehrten Investitionen sowie höheren öffentlichen Ausgaben und sei ein Instrument, die wirtschaftliche Entwicklung in den am wenigsten entwickelten Ländern anzukurbeln. Trotz zahlreicher Teilschuldenerlasse wurde in den Jahren 1996 bzw. 1999 durch den Internationalen Währungsfonds (IWF) und die Weltbank die HIPC-Initiative $^{2}$ ins Leben gerufen, bei der erstmals auch die multilateralen Finanzinstitutionen miteinbezogen wurden. Im Jahr 2005 wurde von den G8-Staaten der nächste Schuldenerlass beschlossen. Dieser Multilaterale Schuldenerlass (MDRI) ${ }^{3}$ sieht teilweise sogar eine 100prozentige Streichung gegenüber den großen multilateralen Gläubigerinstitutionen vor. Innerhalb beider Entschuldungsinitiativen wurden bisher 33 der ärmsten Länder Schulden in Höhe von etwa 170 Mrd. USD erlassen. ${ }^{4}$ Die Tatsache, dass nach knapp zwei Jahrzehnten des Schulden- bzw. Teilschuldenerlasses weiterhin neue Initiativen für hoch verschuldete arme Länder gegründet werden, lässt Zweifel daran aufkommen, ob die erhofften positiven Effekte und gesteckten Ziele eines Schuldenerlasses - Wirtschaftswachstum, Armutsreduzierung und bessere Regierungsführung - überhaupt erreicht werden können.

Stefan Hohberger, Diplom-Volkswirt, wissenschaftlicher Mitarbeiter des Lehrstuhls VWL I der Universität Bayreuth. E-mail: stefan.hohberger@uni-bayreuth.de William Easterly, Debt Relief, Foreign Policy No. 127 (2001), S. 20-26.

2 HIPC steht für Heavily Indebted Poor Countries. Die Schuldeninitiative wurde 1999 beim G7Gipfel in Köln modifiziert, nachdem sich in den ersten drei Jahren der Initiative nur sieben Länder für einen Erlass qualifizierten. Zusätzliche Informationen unter: <http://www.imf.org/external/np/ exr/facts/hipc.htm>.

MDRI steht für Multilateral Debt Relief Initiative: <http://www.imf.org/external/np/exr/facts/ mdri.htm>.

4

Vgl. IDA/IMF (International Development Association and International Monetary Fund), Heavily Indebted Poor Countries (HIPC) Initiative and Multilateral Debt Relief Initiative (MDRI) - Status of Implementation, Washington D. C. 2008, S. 3. 
Um dies ökonomisch beantworten und Aussagen über deren Potential machen zu können, müssen die Schuldenerlassprogramme auf Anreizkompatibilität geprüft werden, vor dem Hintergrund der in den ärmsten Ländern vorherrschenden institutionellen Rahmenbedingungen.

Im vorliegenden Beitrag wird nicht explizit auf die Gründe der hohen Staatsverschuldung sowie die unterschiedlichen Entschuldungsinitiativen eingegangen. Es wird stattdessen versucht, die durch die bisherigen Schuldenerlassprogramme geschaffenen Anreizstrukturen und daraus resultierende Probleme darzulegen und Anreizmechanismen aufzuzeigen, die möglicherweise geeignet wären, eine nachhaltige Schuldensituation der Entwicklungsländer zu erreichen.

\section{A. Status Quo des Schuldenerlasses - Fortschritt oder Stillstand?}

Während frühere Schuldenerlassprogramme in erster Linie an makroökonomische Strukturanpassungsmaßnahmen gekoppelt waren, zielt die HIPC-Initiative ${ }^{5}$ auf die Armutsreduzierung und die institutionelle Dimension wirtschaftlicher Entwicklung. Interessant ist, ob zehn Jahre nach der modifizierten HIPC-Initiative die Entschuldungen zur Erreichung der gesetzten Ziele beigetragen haben oder ob die Lage in den betroffenen Ländern unverändert geblieben ist?

\section{Auswirkungen der Schuldenerlasse auf die HIPC-Länder}

Knapp 200 Mrd. USD Schuldenerlass zeigen durchaus positive Resultate bei vielen Schuldenindikatoren. So offenbaren das Schuldendienst/Export- und Schuldenstand/BIP-Verhältnis gerade in den letzten Jahren deutliche Rückgänge. ${ }^{6}$ Empirische Analysen über den Verschuldungs-Wachstums-Zusammenhang legen dar, dass durch die Reduzierung des Schuldenstandes ein positiver Wachstumseffekt zu verzeichnen sein müsste. ${ }^{7}$ Das Betrach-

Zum Zeitpunkt der Verfassung der Arbeit (Stand: Januar 2009) waren 41 Länder zur Teilnahme an der HIPC-Initiative berechtigt. Hiervon haben sich 33 für die HIPC-Initiative qualifiziert und den Decision Point erreicht. Von diesen 33 Ländern schlossen insgesamt 23 Länder die Initiative ab, d.h., sie erreichten den Completion Point und erhielten damit eine umfassende Schuldenerleichterung. Für eine Übersicht über den aktuellen Stand der HIPC-Länder siehe: <http://go. worldbank.org/4IMVXTQ090>.

Der Schuldendienst/Export-Quotient konnte in den 23 Completion-Point-Ländern ab dem Jahr 2000 von ca. 18\% um zwei Drittel auf knapp 6\% verringert werden. Er liegt damit erstmals seit über zwanzig Jahren unter dem, von 54 ausgewählten Nicht-HIPC-Ländern. Eine ähnliche Entwicklung zeigt auch der Schuldenstand/BIP-Indikator: Etwa ab dem Jahr 1995 konnte der Schuldenstand, gemessen am BIP, in den 23 bereits abgeschlossenen HIPC-Ländern von 180\% auf knapp 60\% im Jahr 2006 gesenkt werden, während das Verhältnis bei den 18 Pre-CompletionHIPCs annähernd konstant (hoch) blieb (vgl. Global Development Finance online 2008). 
ten der durchschnittlichen jährlichen Pro-Kopf-Wachstumsraten enthüllt jedoch ein anderes Bild: ${ }^{8}$ In den Betrachtungszeiträumen 1995 bis 1999 und 2000 bis 2005 ist in den 23 PostCompletion-Point-Ländern - die bis zum Jahr 2008 im Rahmen der HIPC-Initiative entschuldet wurden - im zweiten Zeitfenster zwar eine Steigerung der durchschnittlichen Wachstumsraten von etwa $0,2 \%$ zur Vorperiode zu erkennen, interessant ist jedoch der Vergleich mit den Nicht-HIPC-Ländern. Diese erwirtschafteten von 2000 bis 2005 ca. 0,5\% höhere Wachstumsraten gegenüber der ersten Periode. Ein Vergleich der Wachstumsraten beider Ländergruppen offenbart, dass die Nicht-HIPC-Länder gegenüber den 23 PostCompletion-Ländern in der zweiten Periode fast $1 \%$ pro Jahr stärker wachsen. Aufschlussreich ist auch die Betrachtung der 18 HIPC-Länder, die die HIPC-Initiative bis zum Jahr 2005 abgeschlossen haben. Lediglich sechs dieser Länder konnten zwischen 2000 und 2005 höhere Wachstumsraten gegenüber der Vorperiode aufweisen. Wie es scheint konnten die meisten Länder trotz immenser Schuldenerleichterungen keine positiven Wachstumseffekte erzielen. ${ }^{9}$

\section{Entwicklung der institutionellen Rahmenbedingungen der HIPC-Länder}

Die institutionellen Rahmenbedingungen sind für die Entwicklung eines Landes von entscheidender Bedeutung. Sie formulieren die Spielregeln eines Staates, die maßgeblich das Handeln und Entscheiden der Individuen beeinflussen. Nach North sind Institutionen „the rules of the game in a society or, more formally, [...] the humanly devised constraints that shape human interactions. In consequence they structure incentives in human exchange, whether political, social, or economic."10 Diese Regeln sollen dazu führen, dass sich die Politik durch Prinzipien selbst bindet und Erwartungssicherheit schafft. Dadurch werden

Working Paper No. 02/69 (2002); Benedict J. Clements et al., External Debt, Public Investment, and Growth in Low-Income Countries, IMF Working Paper No. 03/249 (2003); Andrea F. Presbitero, The Debt-Growth Nexus: a Dynamic Panel Data Estimation, Working Papers from Universita' Politecnica delle Marche (I), Dipartimento di Economia, No. 243 (2005); Tito Cordella et al., Debt Overhang or Debt Irrelevance? Revisiting the Debt-Growth Link, IMF Working Paper No. 05/223 (2005).

Vgl. World Development Indicators 2007.

9 Arslanalp/Henry weisen in diesem Zusammenhang darauf hin, dass die höheren Wachstumsraten Ende der 1990er Jahre auf die Strukturanpassungs- und Reformprogramme der Länder zurückzuführen sind (vgl. Serkan Arslanalp, Peter B. Henry, Helping the Poor to Help Themselves: Debt Relief or Aid, NBER Working Paper No. 10230 (2004). Diese wurden mit Unterstützung von IWF und Weltbank bereits vor dem Schuldenerlass erarbeitet und dienen u.a. als Qualifikationskriterium für die HIPC-Initiative. Uganda beispielsweise erreichte im Mai 2000 als erstes Land den Completion-Point und erzielte in den Folgejahren zwischen 2000 und 2005 nur halb so hohe ProKopf-Wachstumsraten. Zambia generierte in diesem Zeitraum sogar leicht höhere Wachstumsraten, erreichte den Completion-Point hingegen erst 2005.

10

Douglass North, Institutions, institutional change and economic performance, Cambridge 1990, S. 3 . 
sowohl den Privaten als auch der Politik Investitionsanreize in Sach- und Humankapital gegeben, was letztendlich zu mehr Wachstum und Wohlstand führt. ${ }^{11}$ Besonders das Konzept der guten Regierungsführung (Good Governance) spielt in diesem Zusammenhang eine wichtige Rolle. Durch das Fehlen konstitutioneller Schranken und rechtsstaatlicher Kontrolle gibt es in Entwicklungsländern kaum Bemühungen wirksame Verschuldungsgrenzen zu implementieren. Um das institutionelle Setting eines Landes bzw. dessen Qualität zu messen, werden seit Mitte der 1990er Jahre verschiedene Governance-Indikatoren erhoben. ${ }^{12}$ Werden aus diesen Indikatoren grundlegende institutionelle Variablen ${ }^{13}$ im Zeitverlauf betrachtet und hinsichtlich Veränderungen untersucht, ${ }^{14}$ können folgende Ergebnisse resümierend festgehalten werden:

Mehr als die Hälfte der 23 Completion-Point-Länder weisen im Rahmen des „Freiheitsindices" von Freedom House keine positiven Entwicklungen auf. ${ }^{15}$ Ein ähnliches Ergebnis ergibt sich bei Betrachtung des Korruptionsniveaus in den Jahren 2002 bis 2007. Bei den etwas breiter angelegten Governance-Indikatoren von Kaufmann/Kraay/Mastruzzi, die nicht nur die Regeln auf der Verfassungs- oder konstitutionellen Ebene, sondern auch auf der Marktebene abbilden, offenbaren sogar fünf von sechs Kategorien im Zeitverlauf Verschlechterungen gegenüber dem Vergleichsjahr 2000. Diese Indikatoren verdeutlichen, dass es trotz der erweiterten Konditionalität der Schuldenerlassprogramme nur zu geringen Verbesserungen des institutionellen Settings kam. Reformen, die auf Regelsetzung, Einhaltung, Sanktionierung sowie auf die Eindämmung willkürlicher politischer Machtausübung zielen, konnten nicht ausreichend implementiert werden.

Die Zusammenhänge zwischen der Qualität der Institutionen, dem Korruptionsniveau und dem Wirtschaftswachstum eines Landes bestätigen eine Vielzahl von empirischen Analysen. Den negativen Einfluss von Korruption auf das BIP-Wachstum zeigt z.B.: Paolo Mauro, Corruption and Growth, in: The Quarterly Journal of Economics, Vol. 110 (1995), S. 681-712. Kaufmann et al. legen mit Hilfe von sechs aggregierten Governance-Indikatoren den signifikanten Zusammenhang zwischen besserer Governance und höherer Entwicklung dar (vgl. Daniel Kaufmann et al., Governance Matters, World Bank Policy Research Working Paper 2196 (1999).

12

In diesem Beitrag wurden insbesondere drei Indikatoren betrachtet: Der „Freiheitsindex“ von Freedom House <http://www.freedomhouse.org>, der „Corruption Perception Index“ von Transparency International <http://www.transparency.org> und die Governance-Indikatoren von Kaufmann/Kraay/Mastruzzi <http://www.govindicators.org $>$.

Grundlegende institutionelle Variablen sind beispielsweise die Gewährung von Grund- und Eigentumsrechten, die Einhaltung und Gleichbehandlung der Individuen vor dem Recht (rule of law), die Qualität bzw. Effektivität der Regierung, die politische Stabilität oder das Ausmaß der Korruption eines Landes.

Insbesondere Veränderungen von institutionellen Faktoren beeinflussen den Wachstumspfad langfristig (vgl. Martin Leschke, Institutionen, Wohlstand und Wachstum in den 90er Jahren eine Cross-Country-Analyse für 80 Staaten, in: Eger, T. (Hrsg.), Institutionen und wirtschaftliche Entwicklung, Berlin 2003, S. 23-51). House. 
Zahlreiche empirische Arbeiten bestätigen nicht nur die geringe Effektivität, ${ }^{16}$ sie geben auch Aufschluss über die Kriterien der Vergabe eines Schuldenerlasses. ${ }^{17}$ So deutet eine starke Pfadabhängigkeit ${ }^{18}$ darauf hin, dass die Verteilung der Schuldenerleichterungen keinen vernünftigen Vergabekriterien folgte und das den grundlegenden Problemen der Entwicklungsländer - schwache ökonomische Institutionen - zu wenig Beachtung geschenkt wurde.

\section{B. Schaffen Schuldenerlasse die richtigen Anreize für eine nachhaltige Schuldensituation?}

Um Gründe für die schwache Entwicklung zu finden, müssen die bisherigen Schuldenerlassprogramme auf Anreizkompatibilität untersucht werden. Fehlen den Akteuren Anreize, mit den zusätzlichen finanziellen Ressourcen den Nutzen der Gesellschaft zu maximieren, ist es unwahrscheinlich, dass Reformen hinsichtlich Armutsbekämpfung und institutionellem Setting geschaffen und somit nachhaltige Schuldensituationen erreicht werden.

\section{Geberverhalten und Trittbrettfahrerproblematik auf Gläubigerseite}

Das Verhalten der Geberländer prägt durch die hohen finanziellen Transferzahlungen maßgeblich das (Negativ-)Verhalten der Entwicklungsländer. So kam es in den 1990er Jahren aufgrund der bevorzugten Gläubigerstellung von IWF und Weltbank zu einer steigenden Kreditvergabe. Die internationalen Finanzinstitutionen waren immer die Ersten, deren Forderungen bedient wurden. Durch den Wegfall des Ausfallrisikos entstand ein Moralhazard-Verhalten. Dieses nicht-intendierte Resultat stellte sich ein, weil die multilateralen Gläubiger die Konsequenzen ihrer ungehinderten Kreditvergabe nicht selbst tragen mussten, sondern durch die bilateralen und privaten Gläubiger aufgefangen wurden. Im Rahmen der HIPC-Initiative und der MDRI wurden die multilateralen Gläubiger mit in den Entschuldungsprozess integriert und erstmals mit den Kosten eines Schuldenerlasses konfron-

Vgl. Nicolas D. Chauvin, Aart Kraay, What Has 100 Billion Dollars Worth of Debt Relief Done for Low-Income Countries?, Washington D. C. 2005; Pernilla Johansson, Debt Relief, Investment and Growth, Working Paper No. 2008:11, Department of Economics, Lund University, Lund 2008; Andrea Presbitero, Debt Relief Effectiveness and Institution Building, MPRA Paper No. 12597 (2008).

Vgl. William Easterly, How Did Heavily Indebted Poor Countries Become Heavily Indebted? Reviewing Two Decades of Debt Relief, in: World Development 2002, Vol. 30(10), S. 16771696; Eric Neumayer, Is Good Governance Rewarded? A Cross-national Analysis of Debt Forgiveness, in: World Development 2002, Vol. 30(06), S. 913-930; Andreas Freytag, Gernot Pehnelt, Debt Relief and Changing Governance Structures in Developing Countries, in: Jenaer Schriften zur Wirtschaftswissenschaft Nr. 31/2006, Friedrich Schiller-Universität Jena, Jena 2006.

Freytag/Pehnelt zeigen in ihrer Studie u.a., dass ein Land, das in der ersten Periode (1990 bis 1994) einen Schuldenerlass erhalten hat, mit einer Wahrscheinlichkeit von nahe eins, auch in der zweiten Periode (1995 bis 1999) einen Schuldenerlass erhält (vgl. Freytag/Pehnelt, Fn. 17, S. 17). 
tiert, was dem Moral-hazard-Verhalten entgegenwirken sollte. Bei genauerer Betrachtung bezüglich der Finanzierungsmodalitäten fällt jedoch auf, dass ein Großteil der anfallenden Kosten, v.a. für die International Development Association (IDA), wieder von bilateralen Gläubigern getragen wird. ${ }^{19}$ So werden für die IDA die geschätzten Kosten der MDRI über die nächsten 40 Jahre, in Höhe von 37 Mrd. USD, von den G8-Mitgliedsstaaten auf einer Pay-as-you-go-Basis durch Zuschüsse in den IDA Replenishment Fund gedeckt werden. ${ }^{20}$ Die Kosten des IWF werden größtenteils durch Zuschüsse der bilateralen Gläubiger des Pariser Clubs in den HIPC Trust Fund finanziert. ${ }^{21}$ Auf diese Weise kommt es zu einer ineffizienten Allokation der bilateralen Entwicklungsgelder. Solange die multilateralen Institutionen nicht die Kosten und Konsequenzen ihrer Vergabepolitik tragen müssen, kann das Moral-hazard-Problem nicht überwunden werden.

Neben dem Moral-hazard-Verhalten der Bretton-Woods-Institutionen stellt die Trittbrettfahrerproblematik für die Effektivität eines Schuldenerlasses ein weiteres erhebliches Anreizproblem auf Seiten der Gläubiger dar. Unter Trittbrettfahren ist in diesem Zusammenhang das Verhalten von internationalen Gläubigern zu verstehen, die die verbesserte Kreditwürdigkeit der bereits entschuldeten Staaten ausnutzen und neue nicht-konzessionäre Kredite mit erhöhten Zinsen vergeben. ${ }^{22}$ Das bedeutet, dass sich entschuldete Länder zu schlechteren als im Rahmen des Schuldenerlasses vorgesehenen Bedingungen neu verschulden. Diese Quersubvention für opportunistische Gläubiger wurde v.a. durch die Einbindung der multilateralen Finanzinstitutionen in den Entschuldungsprozess hervorgerufen. Gerade durch die beiden aktuellen Entschuldungsinitiativen verbesserten sich die Schuldenindikatoren vieler HIPC-Länder erheblich. Durch den vergrößerten Neuverschuldungsspielraum sind besonders die Länder mit verbessertem Kapitalmarktzugang, wie Ghana und Bolivien, für eine Akkumulation nicht-konzessionärer Schulden anfällig. In diesem Zusammenhang ist das steigende Engagement von Emerging Creditors erwähnenswert. ${ }^{23}$ Neben Brasilien, Indien, Korea und Saudi Arabien zählen China und Kuwait zu den größten neuen Gläubigern von Low Income Countries. Um das Trittbrettfahren einzudämmen, schlägt die IDA einen zwei-gleisigen Ansatz vor: ${ }^{24}$

Vgl. IDA (International Development Association), IDA 15: IDA's long-term financial capacity, Resource Mobilization (FRM), Washington D. C. 2007, S. $2 \mathrm{ff}$.

Vgl. IDA (International Development Association), The Multilateral Debt Relief Initiative: Implementation Modalities for IDA, Washington D. C. 2005, S. $9 f$.

21 Vgl. IDA, Fn. 19, S. 14f.

Vgl. IDA (International Development Association), IDA-Countries and Non-concessional Debt: Dealing with the „Free-Rider“-Problem in IDA14 Grant Recipient and Post-MDRI Countries, Resource Mobilization Department (FRM), Washington D. C. 2006.

Reisen/Ndoye gehen insbesondere auf die unbedachte Kreditvergabe von Emerging Creditors an LICs ein (vgl. Helmut Reisen, Sokhna Ndoye, Prudent versus Imprudent Lending to Africa: From Debt Relief to Emerging Lenders, OECD Development Centre Working Paper No. 268 (2008).

Vgl. IDA, Fn. 22, S. 16ff. 
(1) Eine Verbesserung der Koordination unter den Gläubigern im Rahmen des Debt Sustainability Framework ${ }^{25}$ und

(2) Negativanreize für Schuldnerländer in Form von Nachteilen bei den IDA-Finanzierungen.

Beide Ansätze berücksichtigen jedoch zu wenig die Anreizkomponente. So bestehen zum Einen auf Gläubigerseite keine Anreizmechanismen, das opportunistische Verhalten einzudämmen, da sich das Rahmenwerk des DSF lediglich auf rein appellative Maßnahmen beschränkt. Ferner wird nirgendwo der Anspruch des Gläubigers auf Rückzahlung in Frage gestellt. Zum Anderen setzen die Maßnahmen einzig die Schuldner unter Druck: Anreizmechanismen in Form von Volumenreduzierung und/oder einer Verschlechterung der Kreditbedingungen ${ }^{26}$ der konzessionären IDA-Mittel generieren erst Anreize, die geringeren konzessionären Mittel durch vermehrte nicht-konzessionäre zu substituieren.

\section{Fehlanreize auf Schuldnerseite}

Das Verhalten der Gläubiger führt auch in den Schuldnerländern zu einem Moral-hazardVerhalten: Die verschuldeten Staaten antizipieren das bisherige Geberverhalten und nehmen in der Erwartung, dass neu entstandene Schulden erlassen werden, immer wieder neue Kredite auf. Dieses nicht-intendierte Resultat wurde durch die Kreditvergabepolitik von IWF und Weltbank und den langjährigen Entschuldungsmaßnahmen gefördert. Es erzeugt kontraproduktive Anreize und reduziert die eigenen Entschuldungsanstrengungen der Schuldner. So wurden den HIPC-Ländern allein zwischen 1989 und 1997 insgesamt 33 Mrd. USD Schulden erlassen, während 41 Mrd. USD an neuen Krediten aufgenommen wurden. $^{27}$

Fehlanreize entstanden für Schuldnerländer auch aufgrund der ex ante-Konditionalität in den 1980er und 1990er Jahren, da ein Schuldenerlass von angedachten wirtschaftspolitischen Verbesserungen statt von der tatsächlichen Güte der Wirtschaftspolitik im Status Quo abhängig gemacht wurde. ${ }^{28}$ Dies führt zu zwei Anreizproblemen:

Das DSF dient letztlich dazu, einen Leitfaden für die Darlehensgewährung an LICs zu geben. Es kategorisiert Empfängerländer nach ihrer relativen Schuldenbelastung, bei der sowohl ein Basisszenario zu Grunde gelegt wird, als auch mit Hilfe von „Stresstests“ mögliche externe Schocks berücksichtigt werden. Zusätzlich wird erstmals auch die Qualität der Institutionen sowie der Regierungsführung mit einbezogen. Die festgelegten Schwellenwerte für die verschiedenen Schuldenindikatoren sollen als Grundlage und Orientierung für zukünftige Kreditvergabeentscheidungen herangezogen werden. Für detailliertere Informationen siehe: IMF/World Bank, Applying the Debt Sustainability Framework for Low-Income Countries Post Debt Relief, Washington D. C. 2006.

Eine Verschlechterung der Kreditbedingungen beinhaltet höhere Zinsen bzw. Gebühren oder verkürzte Rückzahlungsfristen.

27 Vgl. William Easterly, The Elusive Quest for Growth: Economists' adventures and misadventures in the tropics, Cambridge 2001, S. 128.

28 Vgl. William Easterly, Fn. 17, S. 1681. 
(1) Die Vergabe von finanziellen Mitteln wurde (nur) an Versprechungen, eine bestimmte Reform durchzuführen, gebunden.

(2) Es wurden diejenigen Länder bevorteilt, die in der Vergangenheit eine besonders schlechte Politik betrieben haben.

Aus (1) folgt, dass aufgrund fehlender institutioneller Schranken und funktionierender Kontrollmechanismen in den ärmsten Ländern Anreize entstehen, gegenüber dem IWF bestimmte Reformen lediglich zu versprechen. ${ }^{29}$ So vergaben die Weltbank und der IWF in den 1980er und 1990er Jahren die unter dem Begriff Adjustment lending bekannt gewordenen (Struktur-)Anpassungskredite für bestimmte Reformen. Einer Vielzahl von Ländern wurden mehr als zehn adjustment loans innerhalb von fünfzehn Jahren vergeben, ohne dass sie eine Verbesserung der wirtschaftlichen Struktur oder höhere Wachstumsraten aufzeigen. ${ }^{30}$ Die ex ante-Vergabe eines Kredites vor der tatsächlichen Reformdurchsetzung führt dazu, dass nur die beabsichtigten und gewollten Reformen durchgeführt werden. „Unless incentives are properly aligned, governments will promise, take the money, and then do what they like." ${ }^{31}$ So versprach beispielsweise Kenia der Weltbank für die Gewährung neuer Kredite fünf Mal die gleiche Landwirtschafts-Reform innerhalb von fünfzehn Jahren. Diese Verhaltensmuster führen in den verschuldeten Staaten zu einem „Zick-Zack-Kurs“ der Politik. Dabei werden Reformen angestrebt und soweit durchgesetzt, bis das Land von den Gebern einen neuen Kredit oder Erlass gewährt bekommt, um dann ihre Bemühungen und Reformvorhaben wieder fallenzulassen.

Wie in Punkt (2) erwähnt, haben diejenigen Länder die größte Chance auf Belohnung für Reformen, die in der Ausgangssituation eine besonders schlechte Politik betrieben haben. Denn ausgehend von dieser Basis sind Verbesserungen relativ einfach. So haben die Verantwortlichen in den ärmsten Ländern Anreize arm zu bleiben und weiterhin eine relativ schlechte Politik zu betreiben. ${ }^{32}$ Auf diese Weise kommen sie einerseits einfacher an die von IWF und Weltbank vergebenen Anpassungskredite, andererseits erhalten sie auch eher Schuldenerleichterungen. Das bestätigen auch die empirischen Erkenntnisse über die Allokation der Schuldenerlasse. Birdsall et al. zeigen, dass arme Länder mit schlechter Politik zwischen 1978 und 1998 mehr finanzielle Leistungen erhielten. ${ }^{33}$ Diese Form von Adverser

29

30

31

32

33

Vgl. Paul Collier, The Bottom Billion - Why the Poorest Countries are Failing and What Can Be Done About It, New York 2007, S. 109.

Vgl. William Easterly, Fn. 27, Kapitel 6 ,The loans that were, the growth that wasn't”.

Paul Collier, Fn. 29, S. 109.

Easterly weist darauf hin, dass die herrschenden Eliten einen starken Anreiz haben, den Zustand dauerhafter Armut gar nicht zu ändern, solange Entwicklungsgelder v.a. dann gezahlt werden, wenn es der Bevölkerung besonders schlecht geht (vgl. William Easterly, The White Man's Burden: Why the West's Efforts to Aid the Rest Have Done So Much Ill and So Little Good, New York 2006, Kapitel 4).

Vgl. Nancy Birdsall et al., Policy Selectivity Forgone: Debt and Donor Behaviour in Africa, in: Addison et al. (Hrsg), Debt Relief for Poor Countries, Hampshire 2004, S. 59-89. 
Selektion bestraft jene Entwicklungsländer, die in der Vergangenheit durch harte Reformanstrengungen ihren Schuldendienst rechtzeitig erbracht haben oder eventuell sogar durch einen Regimewechsel ihre Reformwilligkeit unterstrichen haben. Gerade solche Länder sollten von der internationalen Gemeinschaft unterstützt werden, anstatt sie für ihre Kooperationsbereitschaft zu strafen.

\section{Anreizmechanismen für eine nachhaltige Schuldensituation in Entwicklungsländern}

Das Geber- und daraus resultierende Schuldnerverhalten bedingt Anreizstrukturen, die mit einer nachhaltigen Schuldensituation und der Schaffung eines institutionellen Umfelds für produktive Wettbewerbsprozesse nicht kompatibel sind. Ziel dieses Kapitels soll die Diskussion bzw. Gestaltung institutioneller Arrangements sein, die möglicherweise geeignet wären eine nachhaltige Schuldensituation zu erreichen.

\section{Die Anreizsituation eines Diktators}

Zunächst werden Überlegungen zur Analyse einer Diktatur angestellt, die helfen sollen, das Verhalten der herrschenden Elite bei der Gewährung eines Schuldenerlasses zu erklären. Das folgende Modell knüpft an die Arbeiten von Ronald Wintrobe und Pies/Wockenfuß an, blendet jedoch bestimmte Zusammenhänge bewusst aus, um den Fokus auf die entscheidenden Sachverhalte legen zu können. ${ }^{34}$ Es soll gezeigt werden, wie Machthaber in autokratischen Staaten regieren, und wie diese auf einen Ressourcenzufluss von demokratischen Staaten reagieren. Hierfür wird angenommen, dass ein Diktator als Alleinherrscher fungiert. Das bedeutet, dass er über alle politischen Maßnahmen seines Landes entscheiden kann und sich rational verhält, also das für ihn beste Ergebnis wählt. Das Ziel eines Diktators ist der Erhalt seiner politischen Macht. Um dieses zu verwirklichen kann der Diktator über zwei unterschiedliche Wege politische Macht ausüben: ${ }^{35}$ Einerseits kann er sich die Unterstützung der Bevölkerung verdienen. Hierzu zählen beispielsweise Investitionen in Infrastruktur, Ausgaben in Bildung und Gesundheit sowie die Reduzierung der Armut. Dieser Weg reflektiert das gewünschte Verhalten von Seiten demokratischer Staaten. Diese erste Option soll allgemein als „Bereitstellung öffentlicher Güter“ (ÖG) bezeichnet werden. Die

Vgl. Ronald Wintrobe, The Tinpot and the Totalitarian: An Economic Theory of Dictatorship, in: American Political Science Review 84(2), New York 1990, S. 849-872; Ingo Pies, Christof Wockenfuß $\beta$, Armutsbekämpfung versus Demokratieförderung: Wie lässt sich der entwicklungspolitische Trade-Off überwinden?, Diskussionspapier Nr. 2008-3, Lehrstuhl für Wirtschaftsethik, Martin-Luther-Universität Halle-Wittenberg, Halle 2008.

Im Gegensatz zu Wintrobe und Pies/Wockenfuß wird das Modell an dieser Stelle etwas vereinfacht dargestellt und legt den Schwerpunkt auf die Entscheidungsalternativen eines Diktators. Ausführlichere Modellierungen generieren für die hier interessierende Fragestellung wenig Mehrwert. 
zweite Möglichkeit des Diktators politische Macht auszuüben, reflektiert das unerwünschte Verhalten, indem er sich die Unterstützung der Bevölkerung erkauft oder etwaige Oppositionen unterdrückt. Dies kann durch die Verschwendung von Ressourcen, die Gewährung von Privilegien oder auch durch die Ausübung von politischem Druck ${ }^{36}$ auf die Bevölkerung geschehen. Die zweite Option zur Generierung von Macht wird nachfolgend als „Repression“ $(\mathrm{R})^{37}$ bezeichnet. Die bisherigen Überlegungen lassen sich nun in eine mikroökonomische Terminologie übersetzen: Der Diktator hat Zugriff über die gesamten finanziellen Ressourcen des Regimes, dem Staatsbudget B. Dieses teilt er bei gegebenen Kosten auf die konkurrierenden Alternativen ÖG und R so auf, dass seine produzierte Macht möglichst groß ausfällt. ${ }^{38}$ Grafisch lässt sich diese Optimalitätsbedingung der Machtproduktion gemäß Abbildung 1a darstellen.

Die Machtisoquante $\pi^{*}$ repräsentiert das Austauschverhältnis zwischen den Optionen ÖG und $\mathrm{R}$ bei der Produktion von Macht. Die Budgetgerade B stellt die finanzielle Restriktion dar, und spiegelt alle Kombinationsmöglichkeiten der beiden Verhaltensoptionen wider. Im Punkt G erreicht der Diktator das optimale Ergebnis. ${ }^{39}$ Mit dem in diesem Punkt aufgezeigten Verhältnis von ÖG* und $\mathrm{R}^{*}$ produziert der Diktator die größtmögliche Macht. Interessant ist, wie der Diktator auf einen Ressourcenzufluss reagiert, hier speziell in Form eines Schuldenerlasses, und welche Auswirkungen dies auf die beiden Machtausübungsoptionen hat. Ein Schuldenerlass führt zu einer Ausweitung der Budgetrestriktion. ${ }^{40}$ Grafisch (vgl. Abb. 1b) bedeutet das eine Rechtsverschiebung der Budgetgerade auf $\mathrm{B}_{1}$. Der rationale Akteur sieht sich veranlasst, auf die Ausweitung seines finanziellen Spielraums zu reagieren. Um sein neues (höheres) Machtniveau $\pi_{1}$ am kosteneffizientesten $\mathrm{zu}$ erreichen (im Punkt $\mathrm{G}_{1}$ ), kommt es zu einer Ausweitung beider Machtausübungsoptionen auf $\mathrm{Ö}_{1}$ und $\mathrm{R}_{1}$. Welche Tendenzaussagen lassen sich nun aus diesem Modell einer Diktatur ableiten? Eine Ausweitung der finanziellen Ressourcen zieht auf der einen Seite eine

Damit ist u.a. die Abschaffung, Änderung oder Nichteinhaltung von Grundrechten, wie politische und bürgerliche Freiheit gemeint. Ein weiterer wichtiger Punkt sind die Investitionen zur Unterstützung des Militärs, um potentielle Gegner des Regimes zu unterdrücken und so evtl. Revolten zu verhindern.

Der Begriff „Repression“ wurde von Wintrobe übernommen: „Repression includes restrictions on the rights of citizens to criticize the government, the freedom of the press, and the rights of opposition parties to campaign against the government, and the outright prohibition of groups, associations, or political parties opposed to the government" (Ronald Wintrobe, Fn. 34, S. 851). Auf die formale Schreibweise soll hier verzichtet werden.

Der Punkt G entspricht der Minimalkostenkombination der Produktionstheorie, d.h., diejenige Kombination der Produktionsfaktoren, mit der am kostengünstigsten der größte Output generiert wird. früheren Kredite durch der Erlass der Schulden ex post den Charakter von Entwicklungshilfe (grants) an. 


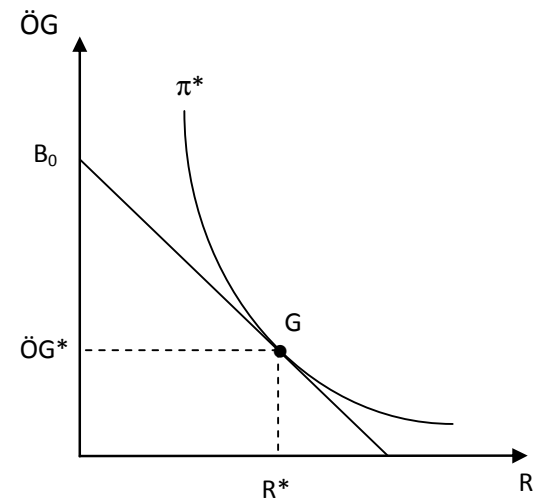

(a)

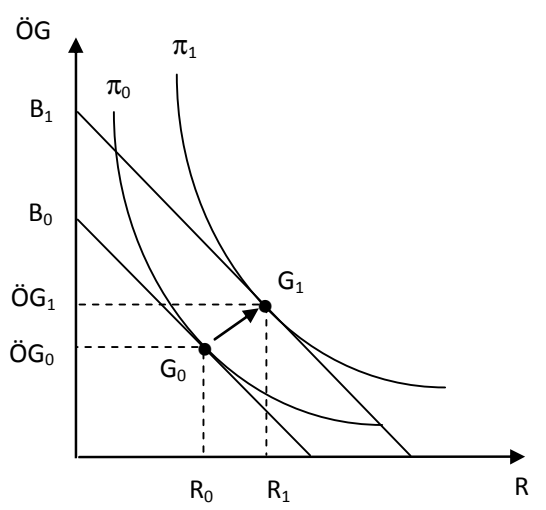

(b)

Abbildung 1: Verhalten eines Diktators bei Ausweitung der Ressourcen (Schuldenerlass)

Quelle: Eigene Darstellung in Anlehnung an Wintrobe (1990) und Pies/Wockenfuß (2008).

höhere Bereitstellung öffentlicher Güter nach sich. Auf der anderen Seite wird aber auch vermehrt Repression ausgeübt. Das bedeutet, dass ein Großteil der Mittel zur Erhöhung von Privilegien und für Korruptionszwecke abgezweigt bzw. allgemein eine schlechte Politik betrieben wird. Folglich führt dies zu einer Festigung der politischen Macht des Diktators.

In diesem Zusammenhang ist es aufschlussreich, auf die Unterschiede zwischen Diktaturen und Demokratien einzugehen. ${ }^{41}$ Geht man davon aus, dass sich ein demokratisch gewählter Politiker zur Ausübung seiner Macht die Unterstützung der Bevölkerung durch Bereitstellung öffentlicher Güter erwerben muss, so bleibt in diesem Modellrahmen als einziges Unterscheidungskriterium das Repressionsniveau. In Demokratien existieren institutionelle Schranken in Form von Regeln und Normen, die bei Nichteinhaltung entsprechend sanktioniert werden. So ist es Politikern in Demokratien gerade nicht möglich, zur Machtsicherung Repression einzusetzen. Je wirkungsvoller die konstitutionellen Beschränkungen der Repressionsmöglichkeiten, umso weniger ist es Diktatoren möglich, in unbegrenztem Umfang Repression auszuüben.

Anhand dieses Modells lassen sich wichtige Schlussfolgerungen ableiten: Erstens besteht für autokratische Regime ohne institutionelle Schranken ein Anreiz, zur Ausübung der politischen Macht Repression einzusetzen. Dieser Anreiz generiert weder Erwartungssicherheit, noch einen angemessenen Rahmen für wachstumsfördernde Investitionen, wo- 
durch letztendlich auch kein aufholender Wachstumsprozess in Gang gesetzt wird. Zweitens führt eine Ausweitung des finanziellen Spielraums eher zu einer Festigung der Strukturen. Und drittens sollte eine Konditionierung der finanziellen Mittel an einem Abbau des Repressionsniveaus anknüpfen.

\section{Trade-Off zwischen Schuldenreduktion und guter Regierungsführung}

Mit Hilfe dieses Modells kann gezeigt werden, dass die Knüpfung eines Schuldenerlasses an Bedingungen wie Reduzierung der Armut - lediglich gemessen am Anteil der Staatsausgaben in Bildung und Gesundheit an den Gesamtausgaben - im Hinblick auf eine langfristig nachhaltige Schuldensituation nicht zielführend ist. Wie das Modell erkennen lässt, bestehen zwar Anreize, einen Teil der zusätzlichen finanziellen Mittel in die gewünschte Verwendungsrichtung zu investieren, dies dient jedoch lediglich dazu, den demokratischen Staaten zu zeigen, dass die auferlegten Konditionen eingehalten werden. ${ }^{42}$ Auf diese Weise steigen die Chancen, auch künftige Schuldenerleichterungen und neue Kreditgewährungen zu erhalten. Eine weitere Ausweitung des finanziellen Spielraums würde in Staaten ohne adäquate institutionelle Regeln zu persistenten Rahmenbedingungen und zu einer Festigung des Regimes führen. Es unterbleiben für die wirtschaftliche Entwicklung grundlegende institutionelle Reformen: „Although debt relief is done in the name of the poor, the poor are worse off if debt relief creates incentives to delay reforms necessary for growth." ${ }^{43}$ Es besteht somit ein Trade-Off zwischen der Schuldenreduktion bzw. einer nachhaltigen Schuldensituation und dem Prinzip der Good Governance. Das bedeutet, dass die Erreichung des einen Ziels nur auf Kosten des anderen erlangt werden kann. Zur Überwindung dieses Trade-Offs ist eine gezielte Veränderung der Rahmenbedingungen erforderlich. Es müssten Anreize geschaffen werden, die die entstanden Fehlanreize auf Gläubiger- und Schuldnerseite überwinden. Nur wenn sich die Politik an bestimmte Regeln bindet und willkürliches Handeln einschränkt, werden institutionelle Rahmenbedingungen geschaffen, die nicht nur Grundlage wirtschaftlichen Wachstums sind, sondern langfristig auch eine nachhaltige Schuldensituation generieren.

"Economic institutions encouraging economic growth emerge when political institutions allocate power to groups with interests in broad-based property rights enforcement, when they create

So konnte in den letzten Jahren, durch die Umsetzung der HIPC-Initiative und die daraus frei werdenden finanziellen Ressourcen, ein Anstieg der Ausgaben für Armutsreduzierung, gemessen am BIP, seit Ende der 1990er Jahre von 7\% auf etwa 9\% im Jahr 2007 verzeichnet werden (vgl. $I D A / I M F$, Fn. 4, S. 6f). Dennoch konnte weder eine signifikante aufholende Entwicklung, noch eine Verbesserung der Regierungsführung beobachtet werden.

William Easterly, Fn. 17, S. 1692. 
effective constraints on power-holders, and when there are relatively few rents to be captured by power-holders." 44

\section{Institutionelle Arrangements für eine nachhaltige Schuldensituation}

Im folgenden Kapitel soll ein möglicher Ordnungsrahmen für eine nachhaltige Schuldensituation skizziert werden. Bevor das Augenmerk auf die Überwindung der Fehlanreize auf Schuldnerseite gelegt wird, ist es wichtig auch die Trittbrettfahrerproblematik auf Gläubigerseite aufzugreifen. Solange die Vergabe von neuen Krediten und das Ausnutzen der verbesserten Zahlungsfähigkeit durch neue Gläubiger nicht eingedämmt werden kann, gerät das Ziel einer nachhaltigen Schuldensituation in Gefahr und bisherige Entschuldungsprogramme würden für die beteiligten Gläubigerländer ,,versunkene Kosten“ darstellen.

\section{1. Überwindung der Trittbrettfahrerproblematik auf Gläubigerseite}

Da die vorgeschlagenen IDA-Ansätze zur Lösung des Trittbrettfahrerproblems hauptsächlich die Schuldnerländer durch Negativanreize unter Druck setzen, sollte eher versucht werden, auf Gläubigerseite anzusetzen. Eine mögliche Variante, die Anspruchsgrundlage der Gläubiger auf Rückzahlung der Kredite einzuschränken und dadurch die Kosten des Trittbrettfahrens zu erhöhen, könnte die bereits seit langer Zeit diskutierte Einführung eines internationalen unabhängigen Insolvenzverfahrens sein. ${ }^{45}$ Die Annahme, dass Staaten nicht „pleite“ gehen können, ist spätestens seit 1982 nichtig, als Mexiko als erstes Entwicklungsland seine Zahlungsunfähigkeit erklärte. Bereits Adam Smith sprach in seinem Werk „The Wealth of Nations“ von einer möglichen Insolvenz verschuldeter Staaten: ${ }^{46}$

„When it becomes necessary for a state to declare itself bankrupt, in the same manner as when it becomes necessary for an individual to do so, a fair, open, and avowed bankruptcy is always the measure which is both least dishonourable to the debtor, and least hurtful to the creditor."

Raffer plädiert beispielsweise für ein Verfahren in Anlehnung an Kapitel 9 des amerikanischen Insolvenzrechts. ${ }^{47}$ Dieses ermöglicht Stadtverwaltungen, die zahlungsunfähig sind, ein Insolvenzverfahren, bei dem die wesentlichen Dienstleistungen für die Bevölkerung garantiert bleiben. Das bedeutet bei Anwendung auf Staatenebene, dass finanzielle Mittel,

Daron Acemoglu et al., Institutions as the Fundamental Cause of Long-Run Growth, in: Aghion (Hrsg.), Handbook of Economic Growth, Vol. 22, Amsterdam 2005, S. 386.

Vgl. hierzu: Kunibert Raffer, Applying Chapter 9 Insolvency to International Debts: An Economically Efficient Solution with a Human Face, in: World Development 1990, Vol. 18(2), Amsterdam 1990, S. 301-311.

Adam Smith, An Inquiry into the Nature and Causes of the Wealth of Nations, Cannan (Hrsg), 5. Auflage, 2000, Library of Economics and Liberty, <http://www.econlib.org/library/Smith/ smWN22.html>. 
die zur Gewährleistung sozialer Mindeststandards ${ }^{48}$ notwendig sind, im Rahmen des Schuldnerschutzes dem Zugriff der Gläubiger entzogen wären. Ohne im weiteren Verlauf der Arbeit auf eine detailliertere Ausgestaltung des Verfahrens einzugehen, soll nachfolgend untersucht werden, ob ein solches Verfahren in der Lage wäre, die Trittbrettfahrerstrategie zu überwinden. Um entsprechende Anreizeffekte bewirken zu können, müssten die Schulden gegenüber allen Gläubigern - sei es multilateraler, bilateraler oder privater Art gleich behandelt werden. Auf diese Weise wäre sichergestellt, dass es keine bevorzugten Gläubiger gibt und die Kosten der Forderungsausfälle auf alle Gläubiger verteilt würden. Bestünde die Möglichkeit einer Zahlungsunfähigkeitserklärung des Schuldnerlandes, hätte jeder Gläubiger anteilige Kosten in Form von Abschreibungen zu tragen. ${ }^{49}$ Wird die Möglichkeit eines Ausfallrisikos und die dadurch entstehenden Abschreibungskosten in die individuelle Kosten-Nutzen-Analyse eines Gläubigers einbezogen, entsteht ein Anreiz, von seiner Strategie abzuweichen. Dieser Anreiz wird stärker, je höher der bereits akkumulierte Schuldenstand des Schuldnerlandes ist. Denn dadurch steigt die Ausfallwahrscheinlichkeit und umso höher werden die erwarteten Kosten eines Forderungsausfalls. Das bedeutet für einen rational handelnden Akteur, dass es sich, mit zunehmenden Schuldenstand und somit höheren erwarteten Kosten eines möglichen Kreditausfalls, ab einem bestimmten Zeitpunkt nicht mehr lohnt, die Strategie des Trittbrettfahrens zu wählen. Eine Kreditvergabe der Gläubiger würde sich demnach enger an dem Schuldenstand eines Landes orientieren. Ein derartiges Arrangement eines unabhängigen und transparenten Schiedsverfahrens würde durch die Gleichbehandlung aller Schulden auch dem Moral-hazard-Verhalten der multilateralen Finanzinstitutionen entgegenwirken. Ein wichtiger Punkt, der bei einer institutionellen Ausgestaltung bedacht werden sollte, ist die Beachtung bereits gewährter Schuldenerleichterungen. Es dürfen nicht diejenigen Länder benachteiligt werden, die bereits an einer Schuldenerlass-Initiative teilgenommen haben. Das bedeutet, wenn sich ein Schuldnerland nur kurze Zeit nach einem Schuldenerlass als zahlungsunfähig erweist, ${ }^{50}$ müssten diejenigen Gläubiger, die ohne Beteiligung an der Initiative weiterhin Kredite vergeben haben, mit einem geringeren Rückzahlungsanspruch ausgestattet werden. Wichtig hierfür wären eine höhere Transparenz und detaillierte Informationen über geplante und vollzogene nicht-konzessionäre Kreditaufnahmen der betroffenen Länder, ähnlich wie das von der Weltbank eingeführte Debtor Reporting System (DRS). Je geringer die Beteiligung an einem Schuldenerlass war, desto geringer ist der Rückzahlungsanspruch und umso höher

Zu den sozialen Mindeststandards eines Landes zählen z.B. Primärbildung, Basisgesundheitsversorgung, Ernährungssicherung, etc.

Zusätzlich würde, unter Einbeziehung des Rechts der Gläubiger auf Rückzahlung und des Schuld50 nerschutzes, ein vom unabhängigen Schiedsgericht erarbeiteter Rückzahlungsplan entworfen.

Hier wäre beispielsweise an ein Land zu denken, dass trotz Teilnahme und Gewährung eines Schuldenerlasses im Rahmen der HIPC-Initiative nach kurzer Zeit bereits wieder Schulden angehäuft hat. 
wäre der Anteil des Landes an den anteiligen Abschreibungskosten. Somit würden Anreize geschaffen, sich entweder an einer Entschuldungsinitiative zu beteiligen oder die Kreditvergabe stärker an den Schuldenindikatoren zu orientieren. ${ }^{51}$

Auch wenn die detaillierte Ausgestaltung eines unabhängigen und transparenten Schiedsgerichts hier offen bleibt, könnte ein derartiges Arrangement durchaus in der Lage sein, das Moral-hazard-Verhalten auf multilateraler Seite und das Trittbrettfahrerproblem auf bilateraler und privater Seite zumindest besser als bisher zu überwinden.

\section{Anreizmechanismen auf Schuldnerseite}

Wichtig ist die Etablierung systematischer Anreizmechanismen mit Hilfe geeigneter Arrangements, die die entstandenen Fehlanreize überwinden können. Allein das durch die fortwährenden Entschuldungsinitiativen hervorgerufene Moral-hazard-Verhalten stellt die Effektivität zukünftiger Schuldenerleichterungen in Frage: ${ }^{52}$

„A once-and-for-all program is greatly superior to a gradual program of increasing relief. The once-and-for-all program has to attempt to establish a credible policy that debt relief will never again be offered in the future, and that it is only giving debt relief to governments with a shift in development orientation. If this is problematic, then the whole idea of debt relief is problematic."

Die Frage ist jedoch: Kann nach zwei Jahrzehnten ein letzter einmaliger Schuldenerlass glaubhaft durchgesetzt werden? Auch wenn dies den verschuldeten Staaten angedroht wird, bleiben weiterhin Anreize bestehen, die bisherige Bereitwilligkeit vieler bilateraler Gläubiger auszunutzen und neue Schulden anzuhäufen. Da auch ökonometrische Analysen größtenteils keine signifikante Evidenz positiver Effekte eines Schuldenerlasses auf das Wirtschaftswachstum erkennen lassen, sollte im Umgang mit weiteren Entschuldungen ein Umdenken stattfinden. Hierzu wäre mein Vorschlag, den entschuldeten Staaten glaubhaft zu signalisieren, dass es nach dem vollständigen Durchlaufen der HIPC-Initiative und des Multilateralen Schuldenerlasses in Zukunft keine weiteren Entschuldungsprogramme geben wird. ${ }^{53}$ Die Aufgabe demokratischer Staaten sollte in Zukunft darin liegen, den entschulde-

Für ein derartiges Arrangement ist ein klar definierter Ordnungsrahmen aufzustellen, wobei einige offene Fragen zu diskutieren sind. So bleibt beispielsweise zu klären, ab welchem Schuldenstand ein Land zahlungsunfähig ist bzw. welcher gängige Schuldenindikator dafür herangezogen wird oder ob sogar ein neuer Indikator erstellt werden sollte. Desweiteren wäre die Höhe bzw. Berechnung der sozialen Mindeststandards, in Abhängigkeit der Bevölkerungszahl, zu definieren. Außerdem ist die Kompatibilität des Verfahrens mit den Anreizstrukturen der Schuldnerländer wichtig. Es dürfen keine Fehlanreize für die Schuldner entstehen, sich bewusst mit dem Gedanken zu verschulden, dass nach einem Insolvenzantrag nur ein Teil zurückgezahlt werden muss.

52 William Easterly, Fn. 17, S. 1692.

53 Selbstverständlich sollten die verbleibenden Länder, die den Completion-Point noch nicht erreicht haben, die aktuellen Programme abschließen dürfen. Auch die Erstellung und Implementierung der länderspezifischen Armutsbekämpfungspapiere (Poverty Reduction Strategy Paper) für diese HIPC-Länder sollten weiterhin vorangetrieben werden. 
ten Staaten Anreize zu bieten, die verbesserte Situation für ihre Entwicklung zu nutzen. Die Gewissheit, dass es nach den Entschuldungsinitiativen der letzten Jahre keine weiteren gibt, sollte für arme Länder keine Bestrafung darstellen. Vielmehr könnte mit Hilfe positiver Anreizmechanismen versucht werden, den bestehenden Trade-Off zu überwinden. Genauer gesagt: Es sollten den Entwicklungsländern Anreize geboten werden, durch eine Verbesserung der Regierungsführung eine nachhaltige Schuldensituation zu erreichen. Das bedeutet, dass ein Land, das bereit ist die institutionellen Rahmenbedingungen und die Regierungsführung zu verbessern durch Zuwendung finanzieller Ressourcen belohnt wird. Ideal wäre, wenn zusätzlich eine Art Wettbewerb um die finanziellen Ressourcen etabliert werden könnte, so dass die einzelnen autokratischen Staaten einen Anreiz haben, durch Kooperation, im Sinne von Good Governance, mehr Geld abzugreifen. Bei der Messung der Regierungsführung sollte beachtet werden, dass nicht die Staatsform Demokratie zwingend das Ziel sein muss, denn dies wird bereits seit vielen Jahren versucht, den afrikanischen Staaten top-down aufzuoktroyieren. Vielmehr sollte untersucht werden, ob sich Formen der Rechtsstaatlichkeit etabliert oder verbessert haben, ob politische und bürgerliche Rechte gewährt werden, ob Korruption abgebaut wird und/oder ob sich die Regierung bzw. herrschende Elite durch Bindungsmechanismen in ihren willkürlichen Handlungen einschränkt. Um die Verbesserung der Regierungsführung zu erfassen, würden sich beispielsweise die Governance-Indikatoren von Kaufmann/Kraay/Mastruzzi oder der Freiheitsindex von Freedom House anbieten. Zur Verhinderung einer „Zick-Zack-Politik“ sollte ein Zurückfallen bzw. eine Verschlechterung der Regierungsführung mit entsprechenden Sanktionen geahndet werden.

Nachfolgend wird versucht, einen Ordnungsrahmen aufzuzeigen, der die bisherigen Überlegungen berücksichtigt: ${ }^{54}$

(1) Eine regierungsunabhängige Institution übernimmt die Verwaltung der finanziellen Mittel und ist für die Allokation der Gelder verantwortlich.

(2) Die Aufbringung der finanziellen Mittel für dieses Arrangement wird folgendermaßen geregelt: Von den jährlichen ODA-Zahlungen der im DAC vertretenen Staaten wird ein Teil in einen neuen Topf abgezweigt. Während im Jahr 2007 von allen DAC-Ländern etwa 104 Mrd. USD (im Durchschnitt ca. 0,3\% des jeweiligen BIP) an Entwicklungshilfe aufgebracht wurden, werden künftig ein Drittel, hier etwa 35 Mrd. USD, auf einen Fonds eingezahlt, verzinst, und im nächsten Jahr zur Ausschüttung bereitgestellt. ${ }^{55}$

Das hier erarbeitete Arrangement wurde in Anlehnung an das Modell von Pies/Wockenfuß zur Überwindung eines Trade-Offs zwischen Armutsreduzierung und Demokratieförderung entwickelt (vgl. Pies/Wockenfuß, Fn. 34, S. 22ff).

55

Eine weitere Möglichkeit, bei der Aufbringung der finanziellen Mittel die Trittbrettfahrerstrategie demokratischer Staaten zu überwinden, modelliert im Vergleich hierzu Pies/Wockenfuß: In ihrem Modell können die in der OECD vertretenen Staaten auf dem Konto einer Stiftung Geldbeträge hinterlegen. Die Stiftung ermittelt dann, welches Zahlungsmitglied den geringsten Betrag (in Prozent seines BIP) hinterlegt hat. Von jedem Land wird dann genau dieser Anteil in einen Fonds 
(3) Aktueller Stand, Beiträge und Umsätze des Fonds, die Verteilungsformel unter Punkt (4) sowie alle Auszahlungsregeln unter Punkt (5) werden von der Institution veröffentlicht und sind für jeden einsehbar.

(4) Nach einer jährlichen Evaluation der Regierungsführung wird das generierte Fondsvolumen von der Institution nach folgender Formel an die Länder ausgeschüttet:

$$
a_{i}=\frac{\left(\frac{V_{t}^{i}-V_{t+1}^{i}}{V_{t}^{i}}\right)-M W_{V}+\left(\frac{V_{t}^{i}-V_{t+1}^{i}}{V_{t}^{i}}\right)^{\beta}}{\sum_{i=0}^{n}\left[\left(\frac{V_{t}^{i}-V_{t+1}^{i}}{V_{t}^{i}}\right)-M W_{V}+\left(\frac{V_{t}^{i}-V_{t+1}^{i}}{V_{t}^{i}}\right)^{\beta}\right]} \cdot F
$$

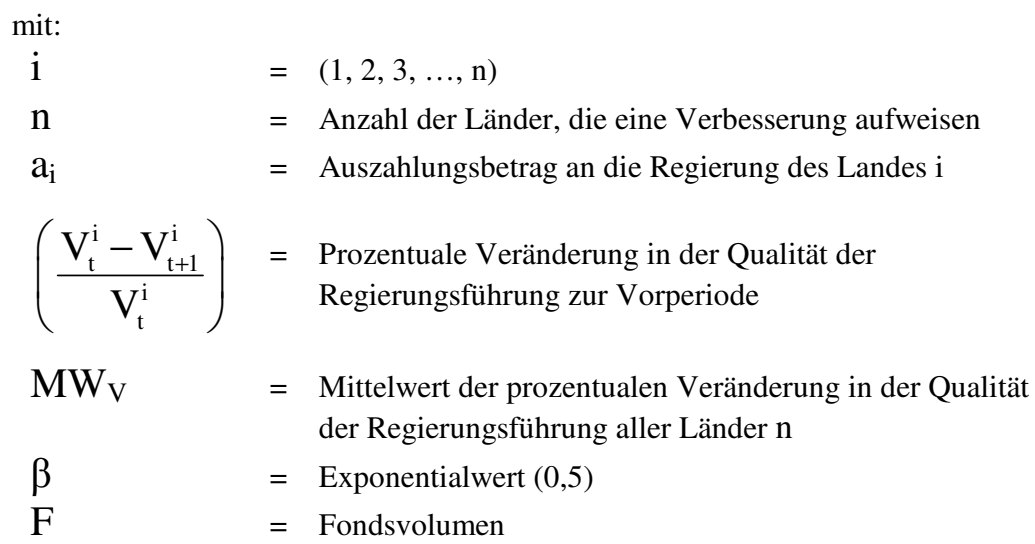

(5) Für eine Auszahlung sind teilnahmeberechtigt: Alle Entwicklungsländer, die in mindestens einer der beiden Kategorien (politische Rechte und/oder bürgerliche Freiheiten) des Freiheitsindex von Freedom House ${ }^{56}$ mit (nur) „teilweise frei“ bewertet sind. $\mathrm{Zu}$ Beginn wird für jedes Entwicklungsland der aktuelle Status Quo bestimmt. Jedes Land,

weitergleitet. Die restlichen Mittel werden an die jeweiligen Regierungen wieder zurückgezahlt (vgl. Pies/Wockenfuß, Fn. 34, S. 22f). Verbesserung der Regierungsführung bietet sich an, da er mit insgesamt 25 Fragen in 7 Unterkategorien ein breites Spektrum von institutionellen Regeln umfasst. 
das sich in dem Index zwischen 7 (nicht frei) und 2,5 (frei) ${ }^{57}$ um mindestens 0,5 Punkte verbessert, hat einen Anspruch auf Auszahlung. Jede Verbesserung um 0,5 Punkte gegenüber dem Status Quo wird nur einmal belohnt. Bei Rückstufung eines Landes geht der Anspruch auf Auszahlung solange verloren, bis das Land den zuletzt erreichten Freiheitsstand wieder erlangt hat.

Welche Anreizwirkungen würden von diesem Arrangement ausgehen? Durch die regierungsunabhängige Institution ${ }^{58}$ (Punkt 1) wird sichergestellt, dass einzelne Länder nicht aus politischen Gründen Zahlungen an bestimmte Länder gewähren. Die Aufteilung der ODA-Zahlungen (Punkt 2) sorgt dafür, dass der Topf der allgemeinen Entwicklungshilfezahlungen abnimmt. Das beinhaltet für die Entwicklungsländer, dass sie zwar weiterhin finanzielle Unterstützung erhalten, diese aber ohne Anstrengung zur institutionellen Verbesserung geringer ausfallen. Punkt (3) führt nicht nur zu Vorteilen einer höheren Transparenz des Verfahrens, sondern signalisiert den Regierungen unterentwickelter Staaten gleichzeitig Erwartungssicherheit über finanzielle Ressourcen, wenn diese zur Kooperation bereit sind. Sobald das Fondsvolumen größer null ist, haben die politischen Akteure einen Anreiz, von ihrem Machtausübungs-Gleichgewicht abzuweichen, da sie auf diese Weise zusätzliche Einnahmen erzielen können. Um diese Anreizmechanismen auf Schuldnerseite nachzuvollziehen, ist die Auszahlungsformel (Punkt 4) näher zu erläutern:

Die Veränderung in der Qualität der Regierungsführung zur Vorperiode wird relativ gemessen. Eine Verbesserung des Freedom House-Index von 7 auf 6 [(7-6)/7 = 1/7] ist auf diese Weise weniger wertvoll als von 5 auf $4[(5-4) / 5=1 / 5]$. Somit wird gleichzeitig ein positiv steigender Zusammenhang zwischen prozentualer Verbesserung und Auszahlungsbetrag modelliert. Um unter den Entwicklungsländern eine Art Wettbewerb zu implementieren, wird die relative Veränderung eines Landes i in Bezug zum Mittelwert $\mathrm{MW}_{\mathrm{V}}$ der positiven Veränderungen aller Länder n gesetzt. Zeigt ein Land eine höhere Verbesserung als der Durchschnitt auf, steigt sein Auszahlungsbetrag. Die Formel enthält noch einen zweiten Wettbewerbsfaktor: Der Nenner spiegelt die Summe der institutionellen Verbesserungen aller Länder n wider. Aufgrund des begrenzten Fondsvolumens, das durch obige Formel immer komplett an die berechtigten Länder ausgeschüttet wird, bedeutet die Verbesserung eines Landes gleichzeitig eine Reduzierung der Auszahlungen aller anderen Länder. Durch diese beiden Mechanismen entsteht für jedes Land ein Anreiz, sich „,besser“ bzw. auf „Kosten der anderen“ zu entwickeln, um einen höheren Anteil am Fondsvolumen

Sobald die Entwicklungsländer als „freies Land“ geratet werden (1 bis 2,5), sind diese für eine Auszahlung nicht mehr berechtigt. Von einem bereits „freien“ Status noch ,freier“ zu werden, würde deren Anteil nur so erhöhen, dass für „schwächere“ Länder weniger übrig bleibt. Dies würde zu geringeren Anreizen für Verbesserungen führen. rungsunabhängig ist. Dass dies prinzipiell möglich ist, zeigt das Beispiel unabhängiger Notenbanken. 
zu erhalten. Der zweite Teil des Zählers sorgt aufgrund des exponentiellen Faktors ${ }^{59} \beta$ dafür, dass Länder für eine höhere prozentuale Heraufstufung innerhalb einer Periode entsprechend mehr belohnt werden. Die Spielregeln in Punkt (5) verhindern, dass die Entwicklungsländer eine „Zick-Zack-Politik“ betreiben, da jede Verbesserung nur einmal berücksichtigt und ein Rückschritt mit einer Auszahlungssperre sanktioniert wird. Bezogen auf das Diktator-Modell verändern sich für Diktatoren, wegen der Möglichkeit bzw. Chance auf zusätzliche finanzielle Ressourcen, die relativen Preise ihrer Machtausübungsoptionen. Rationale Diktatoren haben aufgrund dessen solange einen Anreiz Repression abzubauen und durch Good Governance zu substituieren, bis der erwartete Grenzertrag den erwarteten Grenzkosten entspricht. Die rationalen Diktatoren entscheiden innerhalb ihrer individuellen Kosten-Nutzen-Analyse selbst, welches Governance-Niveau sie wählen. D.h., die nötigen Reformen würden nicht top-down verordnet, sondern bottom-up von den betroffenen Akteuren selbst implementiert. Hinzu kommt, dass gerade Länder mit relativ hohem Verschuldungsgrad - in Abwesenheit zukünftiger Schuldenerlasse - hohe Anreize haben, die Möglichkeit einer finanziellen Zuwendung zu nutzen. Denn die Auszahlungen machen sich in den öffentlichen Haushalten verschuldeter armer Länder vergleichsweise stärker bemerkbar.

Die hier verwendete Verteilungsformel stellt lediglich eine Variante dar, die finanziellen Mittel effektiver zu verteilen, um so mögliche Fehlanreize zu verhindern. Bei genauerer Ausgestaltung des institutionellen Arrangements wären noch offene Fragen zu diskutieren: Zunächst wäre zu klären, welcher Indikator zur Darstellung der „Verbesserung der Regierungsführung" herangezogen werden sollte. Mittlerweile gibt es eine Vielzahl von Governance-Indikatoren, die sich für eine derartige Evaluierung eignen würden. Es wäre von Vorteil, einen möglichst fein skalierten Index heranzuziehen, um eine akkuratere Messung zu erhalten. Eventuell wäre es sogar sinnvoll, mehrere Indizes zu kombinieren. Ein ebenso wichtiger Punkt, den es zu diskutieren gilt, wäre die Aufnahme des Schuldenstandes eines Landes in die Formel. Für Länder mit sehr niedrigem Schuldenstand sollte zusätzlich ein positiver Effekt auf den Auszahlungsbetrag geschaffen werden, während auf der anderen Seite der Auszahlungsbetrag mit zunehmendem Schuldenstand evtl. sogar überproportional abnehmen könnte. Entscheidend wäre, dass die dadurch steigende Gefahr einer Kreditaufnahme bei anderen Gläubigern durch entsprechende Arrangements auch auf Gläubigerseite eingedämmt werden müsste.

\section{Fazit}

Trotz der seit knapp zwei Jahrzehnten gewährten Schuldenerlassprogramme konnten viele Entwicklungsländer ihre Schuldensituation bis heute nicht nachhaltig lösen. Hierfür lassen

Da in prozentualen Größen gerechnet wird, muss der Exponentialfaktor $\beta$ kleiner eins sein, damit ein positiv steigender Zusammenhang modelliert werden kann. Aus diesem Grund wurde der Wert 0,5 gewählt. 
sich zweierlei Gründe finden: Erstens befinden sich die institutionellen Rahmenbedingungen sowie die Regierungsführung der betroffenen Länder nicht nur auf einem sehr niedrigen Niveau, sie zeigen im Zeitverlauf auch größtenteils keine Verbesserungen auf. Zweitens wurden bei den bisherigen Schuldenerlassprogrammen die Anreize auf beiden Marktseiten zu wenig beachtet. Trittbrettfahrerproblematik auf der Gläubigerseite und enorme Fehlanreize auf der Schuldnerseite sind die Auswirkungen einer ungehinderten Kreditvergabepolitik der multilateralen Finanzinstitutionen, einem immer wieder gewährtem Schuldenerlass und einer nicht ausreichenden Orientierung an vernünftigen Vergabekriterien. Diese beiden Entwicklungen schufen Anreizstrukturen, die mit einer nachhaltigen Schuldensituation nur bedingt kompatibel sind. Empirische Arbeiten der letzten Jahre belegen die eingeschränkte Effektivität bisheriger Entschuldungsinitiativen. Hinzu kommt, dass in autokratischen Staaten aufgrund fehlender institutioneller Schranken eine Ausweitung der finanziellen Mittel eher zu einer Erhöhung des politischen Repressionsniveaus in Form von Bad Governance führt. Die Konsequenz ist eine Festigung des diktatorischen Regimes. Die Ziele Schuldenreduktion bzw. Erreichung einer nachhaltigen Schuldensituation und Verbesserung der Regierungsführung stehen in einem Konflikt zueinander. Für die Überwindung dieses Trade-Offs ist ein Umdenken notwendig. Um dem Moral-hazard-Verhalten auf Schuldnerseite entgegenzuwirken, sollten nach dem Abschluss der beiden aktuellen Initiativen vorerst keine weiteren Schuldenerlassprogramme zur Anwendung kommen. Es müsste stattdessen versucht werden, Anreizmechanismen zu schaffen, damit die verbesserte Schuldensituation auch nachhaltig bestehen bleibt. Hierfür wäre die Gestaltung eines institutionellen Ordnungsrahmens nötig, der versucht, das Ziel einer nachhaltigen Schuldensituation durch eine Verbesserung der Regierungsführung zu erreichen. Durch die Gestaltung eines Arrangements, bei dem die Anreizstrukturen sowohl auf Gläubiger-, als auch auf Schuldnerseite beachtet werden, könnten die entstandenen Fehlanreize früherer Schuldenerlassprogramme überwunden und das Ziel einer nachhaltigen Schuldensituation zumindest besser als bisher erreicht werden. Mein Vorschlag wäre die Kombination aus einem unabhängigen und transparenten Schiedsverfahren in Verbindung mit einer Ausgestaltung des beschriebenen institutionellen Ordnungsrahmens. Damit ist auf Gläubigerseite die Trittbrettfahrerstrategie in Folge einer möglichen Zahlungsunfähigkeit weit weniger lohnend, während auf Schuldnerseite institutionelle Reformen bottom-up implementiert werden könnten.

Der in dieser Arbeit aufgezeigte Ordnungsrahmen stellt lediglich einen Versuch dar, die bestehenden (Fehl-)Anreize der bisherigen Schuldenerlassinitiativen in den Blick zu nehmen und mögliche Problemlösungen aufzuzeigen. Für eine tatsächliche Implementierung eines derartigen Arrangements wären zahlreiche offene Fragen, z.B. die Wahl eines geeigneten Governance-Indikators oder die Einbeziehung weiterer Variablen - wie den Schuldenstand - in die Verteilungsformel, zu diskutieren. Wichtig hierbei wäre, dass das Arrangement für beide Marktseiten anreizkompatibel ist. Sicherlich hängt der Erfolg eines solchen Konzepts, institutionelle Reformen bottom-up zu implementieren, auch von dem 
Reformwillen der betroffenen Schuldnerländer selbst ab. Aktuelle Entwicklungen in Afrika zeigen, dass einige Staaten durchaus bereit sind, sich an bestimmte Prinzipien zu binden. So z.B. durch den African Peer Review Mechanism (APRM) innerhalb der NePAD-Initiative. ${ }^{60}$ Der APRM stellt eine Art Informations- und Kontrollmechanismus dar und fußt auf dem Prinzip der freiwilligen Selbstbindung. So sollen sich die afrikanischen Staaten gegenseitig evaluieren um die gesteckten Ziele besser erreichen zu können. Die Verknüpfung eines institutionellen Arrangements mit der unterstützenden Funktion solcher freiwilligen Bindungsmechanismen würde das Potential zur Erreichung einer nachhaltigen Schuldensituation deutlich erhöhen.

\section{Anhang}

Entwicklung der politischen und bürgerlichen Rechte (kombiniert) in 23 Post-CompletionPoint Ländern.

\begin{tabular}{|l|l|l|l|l|l|l|l|l|c|}
\hline & \multicolumn{2}{|c|}{1995} & \multicolumn{2}{|c|}{2000} & \multicolumn{2}{|c|}{2004} & \multicolumn{2}{|c|}{2007} & $\begin{array}{c}\text { Verbesserung im } \\
\text { Zeitablauf? }\end{array}$ \\
\hline Benin & 2 & F & 2 & F & 2 & F & 2 & F & N \\
\hline Bolivia & 3 & PF & 2 & F & 3 & PF & 3 & PF & N \\
\hline Burkina Faso & 4,5 & PF & 4 & PF & 4,5 & PF & 4 & PF & J** \\
\hline Cameroon & 6 & NF & 6,5 & NF & 6 & NF & 6 & NF & N \\
\hline Ethiopia & 4,5 & PF & 5 & PF & 5 & PF & 5 & PF & N \\
\hline Gambia & 6,5 & NF & 6 & NF & 4 & PF & 4,5 & PF & J* \\
\hline Ghana & 4 & PF & 2,5 & F & 2 & F & 1,5 & F & J* \\
\hline Guyana & 2 & F & 2 & F & 2 & F & 2,5 & F & N \\
\hline Honduras & 3 & PF & 3 & PF & 3 & PF & 3 & PF & N \\
\hline Madagascar & 3 & PF & 3 & PF & 3 & PF & 3,5 & PF & N \\
\hline Malawi & 2,5 & F & 3 & PF & 4 & PF & 4 & PF & N \\
\hline Mali & 2,5 & F & 2,5 & F & 2 & F & 2,5 & F & N \\
\hline Mauritania & 6 & NF & 5,5 & NF & 5,5 & NF & 4 & PF & J* \\
\hline
\end{tabular}

60

Die NePAD-Initiative (New Partnership for Africa's Development) wurde 2001 gegründet und umfasst mittlerweile alle 53 Staaten der Afrikanischen Union. Die entscheidende Neuerung liegt nicht in den anvisierten Zielen wie Wirtschaftswachstum und Good Governance, sondern in den Mitteln und Verfahren, mit denen diese Ziele erreicht werden sollen (vgl. Markus Beckmann, NePAD und der African Peer Review Mechanism - Zum Potential politischer Selbstbindung, Diskussionspapier Nr. 2007-8, Lehrstuhl für Wirtschaftsethik, Martin-Luther-Universität HalleWittenberg, Halle 2007). Beckmann gibt auch eine ausführliche Diskussion über Stärken und Schwächen des APRM als kollektiven Bindungsmechanismus. 


\begin{tabular}{|l|l|l|l|l|l|l|l|l|c|}
\hline Fortsetzung & \multicolumn{2}{|c|}{1995} & \multicolumn{2}{|c|}{2000} & \multicolumn{2}{|c|}{2004} & \multicolumn{2}{|c|}{2007} & $\begin{array}{c}\text { Verbesserung im } \\
\text { Zeitablauf? }\end{array}$ \\
\hline Mozambique & 3,5 & PF & 3,5 & PF & 3,5 & PF & 3 & PF & J \\
\hline Nicaragua & 4 & PF & 3 & PF & 3 & PF & 3 & PF & J \\
\hline Niger & 4 & PF & 4 & PF & 3 & PF & 3,5 & PF & J** \\
\hline Rwanda & 6,5 & NF & 6,5 & NF & 5,5 & NF & 5,5 & NF & J \\
\hline Sao Tome \& Principe & 1,5 & F & 1,5 & F & 2 & F & 2 & F & N \\
\hline Senegal & 4,5 & PF & 3,5 & PF & 2,5 & F & 2,5 & F & J* \\
\hline Sierra Leone & 6,5 & NF & 4,5 & PF & 3,5 & PF & 3 & PF & J* \\
\hline Tanzania & 5 & PF & 4 & PF & 3,5 & PF & 3,5 & PF & J \\
\hline Uganda & 4,5 & PF & 5,5 & PF & 4,5 & PF & 4,5 & PF & N \\
\hline Zambia & 3,5 & PF & 4,5 & PF & 4 & PF & 3,5 & PF & N \\
\hline
\end{tabular}

Anmerkungen: Die jeweils linke Zahl zeigt den Umfang der gewährten politischen Rechte und bürgerlichen Freiheiten mit folgender Einteilung an: 1 bis 2,5 „frei“ (F), 3 bis 5 ,teilweise frei“ (PF), 5,5 bis 7 ,nicht frei“ (NF). Das Sonderzeichen * bedeutet, dass sich das Land im Zeitablauf um mindestens 2 Punkte verbessert hat. ** bedeutet, dass im Vergleich zum Ausgangsjahr 1995 zwar eine Verbesserung vorliegt, es aber im Verlauf auch rückläufige Entwicklungen gab.

Quelle: Daten von Freedom House <www.freedomhouse.org>, eigene Darstellung. 